\title{
In vitro coronary flow after transcatheter aortic valve-in-valve implantation: A comparison of 2 valves
}

\author{
Sina Stock, MD, ${ }^{\mathrm{a}}$ Michael Scharfschwerdt, PhD, ${ }^{\mathrm{a}}$ Roza Meyer-Saraei, PhD, ${ }^{\mathrm{a}}$ Doreen Richardt, MD, ${ }^{\mathrm{a}}$
} Efstratios I. Charitos, MD, PhD, ${ }^{\mathrm{b}}$ Hans-Hinrich Sievers, MD, ${ }^{\mathrm{a}}$ and Thorsten Hanke, MD ${ }^{\mathrm{a}}$

\begin{abstract}
Background: Transcatheter aortic valve-in-valve implantation (TAVI-ViV) is an evolving treatment strategy for degenerated surgical aortic valve bioprostheses (SAVBs). However, there is some concern regarding coronary obstruction, especially after TAVI-ViV in calcified SAVBs with externally mounted leaflets. We investigated in vitro coronary flow and hydrodynamics after TAVI-ViV using 2 modern SAVBs with externally and internally mounted leaflets.
\end{abstract}

Methods: Aortic root models including known risk factors for coronary obstruction served for the implantation of SAVBs with either externally mounted leaflets (St Jude Trifecta, size 25) or internally mounted leaflets (Edwards Perimount Magna Ease, size 25). Left and right coronary flow, as well as hydrodynamic parameters, were measured before and after TAVI-ViV with an Edwards Sapien XT transcatheter heart valve, size 23. After the first experimental run, the SAVB leaflets were artificially "calcified," and the measurements were repeated.

Results: In both models, noncalcified and calcified, there was no significant reduction in coronary flow with either the Trifecta or the Perimount Magna Ease SAVB. After TAVI-ViV, in the noncalcified model, the mean pressure gradient was increased (Trifecta, $P=.0001$; Perimount Magna Ease, $P=.006$ ) and the geometric orifice area was decreased $(P<.001$ for both), whereas in the calcified model, the mean pressure gradient was decreased $(P<.001$ for both) and the geometric orifice area was increased $(P<.001$ for both).

Conclusions: In our specific model, in noncalcified as well as calcified conditions, TAVI-ViV is feasible with either SAVB (Trifecta or Perimount Magna Ease) without an increased risk of coronary obstruction. Nevertheless, before clinical application of these results, thorough preoperative assessment, considering the different limitations of this model, is mandatory. ( $\mathrm{J}$ Thorac Cardiovasc Surg 2017;153:255-63)

The transcatheter aortic valve-in-valve implantation technique (TAVI-ViV) is an appealing treatment option for patients with a degenerated surgical aortic valve bioprosthesis (SAVB) and in situations of expected high

\footnotetext{
From the Department of a Cardiac and Thoracic Vascular Surgery, University of Luebeck, Luebeck, Germany; and ${ }^{\mathrm{b}}$ Department of Cardiac Surgery, University of Halle, Halle, Germany.

This work was supported by the German Heart Foundation/German Foundation for Heart Research (Grant number F/30/12).

S.S. and M.S. contributed equally to this work.

Received for publication April 12, 2016; revisions received Sept 22, 2016; accepted for publication Sept 24, 2016.

Address for reprints: Hans-Hinrich Sievers, MD, Department of Cardiac and Thoracic Vascular Surgery, University of Luebeck, Ratzeburger Allee 160, 23538 Luebeck, Germany (E-mail: Hans-Hinrich.Sievers@uksh.de). $0022-5223 / \$ 36.00$

Copyright (c) 2016 by The American Association for Thoracic Surgery http://dx.doi.org/10.1016/j.jtcvs.2016.09.086
}

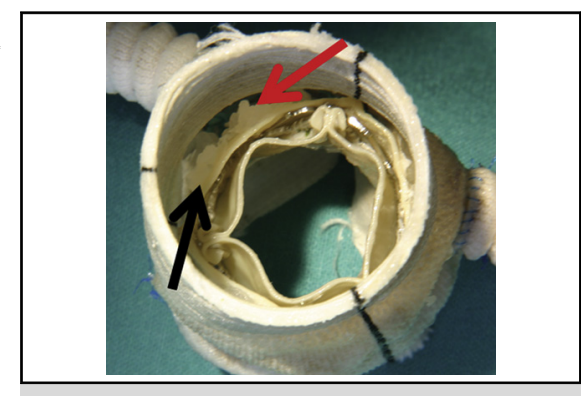

Valve-in-valve implantation of a Sapien XT in a calcified Trifecta surgical aortic valve bioprosthesis.

\section{Central Message}

This limited in vitro study shows no impairment of coronary flow after transcatheter aortic valve-in-valve implantation in surgical bioprostheses with internally or externally mounted leaflets.

\section{Perspective}

Transcatheter aortic valve-in-valve implantation is an emerging treatment for failing bioprostheses, potentially reducing coronary flow, especially in bioprostheses with externally mounted leaflets. This effect was not found in this limited in vitro study comparing bioprostheses with externally and internally mounted leaflets. The limitations of this model must be considered before clinical application.

See Editorial Commentary page 264. surgical risk. However, apart from the numerous advantages compared with redo cardiac surgery, there are concerns about the risk of coronary obstruction as a potential lifethreatening complication. ${ }^{1}$ In the global Valve-in-Valve Registry, this complication has been validated in $3.5 \%$ of patients undergoing TAVI-ViV. ${ }^{2}$ Along with predisposing anatomic factors, such as low coronary ostia height, narrow aortic root, or narrow sinotubular junction, the geometry of

Scanning this QR code will take you to supplemental figure and video for this article. 


\section{Abbreviations and Acronyms \\ $\mathrm{dP}_{\max }=$ maximum pressure gradient \\ $\mathrm{dP}_{\text {mean }}=$ mean pressure gradient \\ GOA $=$ geometric orifice area \\ $\mathrm{LCF} \quad=$ left coronary flow \\ RCF = right coronary flow \\ SAVB = surgical aortic valve bioprosthesis \\ TAVI-ViV $=$ transcatheter aortic valve-in-valve implantation \\ THV $=$ transcatheter heart valve}

the primarily implanted SAVB and its extension of calcification may affect the risk of coronary obstruction. ${ }^{1-5}$

Two different geometric types of modern stented SAVBs are available: those with internally mounted leaflets, such as the Edwards Perimount Magna Ease (Edwards Lifesciences, Irvine, Calif), and those with externally mounted leaflets, such as the St Jude Trifecta (St Jude Medical, St Paul, Minn). The geometry of SAVBs with externally mounted leaflets causes a larger geometric orifice area (GOA) compared with SAVBs with internally mounted leaflets, but the wide extension of leaflet tissue beyond the diameter of the valve's stent may influence coronary flow ${ }^{6}$ (Figure 1).

Gurvitch et $\mathrm{al}^{1}$ described coronary obstruction after TAVI-ViV with a balloon-expandable transcatheter heart valve (THV) (Sapien XT, size 23; Edwards Lifesciences or CoreValve, size 26; Medtronic, Minneapolis, Minn) in 2 patients with highly degenerated SAVB (Sorin Mitroflow, size 21; LivaNova, London, United Kingdom). In these patients, who presented with small aortic roots, the externally mounted leaflets led to closely proximal valve tissue and coronary ostia. The leaflet tissue occluded at least 1 coronary ostium after TAVI-ViV and caused peri-interventional death.

The objective of this in vitro investigation was to identify specific differences in TAVI-ViV using 2 geometrically different SAVBs, in terms of coronary flow as well as hydrodynamic performance, to identify potential coronary obstruction and allow for preinterventional planning to avoid or mitigate this complication.

\section{MATERIALS AND METHODS \\ Valves}

SAVBS. In this in vitro investigation, the SAVB with internally mounted leaflets was the Edwards Perimount Magna Ease $(n=5)$ and the SAVB with externally mounted leaflets was the St Jude Trifecta $(n=5)$, both of size 25. After the first experimental run, leaflet "calcification" was simulated using glue. The leaflet calcification was of alternating thickness, with a maximum thickness of $5 \mathrm{~mm}$.

THV. To perform TAVI-ViV, the balloon-expandable Edwards Sapien XT $(n=2)$, size 23 , was used to achieve optimal hydrodynamics. ${ }^{5,7}$

\section{Aortic Root Models}

The design of the aortic root models was intended to imitate risk factors that are assumed to favor coronary obstruction. Thus, the design was based on an investigation by Ribeiro et $\mathrm{al}^{8}$ revealing a coronary ostia-to-aortic valve annulus distance of $<12 \mathrm{~mm}$ in $86 \%$ of patients with coronary obstruction (mean distance, $10.6 \mathrm{~mm}$ ) and an average sinus of Valsalva diameter of $28.1 \mathrm{~mm}$. We created 2 aortic root models (Figure 2) with different coronary ostia heights $(8 \mathrm{~mm}$ and $10 \mathrm{~mm})$ using aortic sinus prostheses of the smallest available size, $26 \mathrm{~mm}$ (Uni-Graft W Sinus; B. Braun, Melsungen, Germany). Sinus prostheses were used to imitate physiological conditions as close as possible. The nomenclature refers to the diameter of the annulus and sinotubular junction, whereas the diameter of the sinuses is $33 \mathrm{~mm}$. A circular strengthening with felt, situated below the sinuses tissue, simulated the aortic annulus and ensured an identical implantation height of the SAVB. The coronary arteries were simulated by an 8 -mm Dacron prostheses (B. Braun).

\section{Physiological Mock Circulation}

The physiological circulation was imitated by a pulse duplicator that can be adjusted to produce different cardiac output volumes, afterloads, and heart rates, allowing for the evaluation of hydrodynamic parameters. ${ }^{9}$ A camera on top of the aortic root models enabled visual observation and photographs.

\section{Coronary Flow Device}

In vivo, the right coronary flow (RCF) and left coronary flow (LCF) depend on the difference in myocardial flow resistance during a cardiac cycle. In systole, the contraction of the heart muscle leads to greater flow resistance and subsequently less coronary perfusion compared with diastole. This effect is stronger in the left ventricle than in the right ventricle. ${ }^{10}$

To imitate this physiological coronary flow, the Dacron prostheses, simulating the coronary arteries, were connected to the coronary flow device (Figure 3), which consists of 2 sealed power chambers with elastic tubes inside. During systole, the power chambers can be pressurized, leading to compression of the elastic tubes and a subsequent reduction in coronary flow as described above. The power chambers surrounding the right and left coronary artery can be pressurized independently. During diastole, the power chambers are decompressed.

The flow curves for LCF and RCF in our model (Figure 4) are comparable to the physiological coronary flow and support the validity of this coronary flow device. For our experiments, pressurization was guided by a flow-based intraoperative coronary graft patency assessment. ${ }^{10}$

\section{Experimental Procedure}

For each aortic root model, SAVB type and experimental run, we performed 5 measurements (Figure E1), and calculated the mean value. For the first experimental run, the Trifecta or the Perimount Magna Ease SAVB was sewn into an aortic root model, and the conduit was inserted into the mock circulation. ${ }^{9}$ The following parameters were measured: diastolic $\mathrm{LCF}$ and $\mathrm{RCF}$, mean pressure gradient $\left(\mathrm{dP}_{\text {mean }}\right)$, and maximum pressure gradient $\left(\mathrm{dP}_{\max }\right)$. The GOA was identified from photographs taken by the high-speed camera. Subsequently, we explanted the conduit, performed TAVI-ViV with the Sapien XT, commissure-to-commissure with the SAVB, and repeated all measurements (Video 1).

For the second experimental run, we simulated calcification of the SAVB by using glue (Figure 5) and repeated the tests according to the protocol. To simulate leaflet thickening as well as leaflet stiffening, we used 2 glues with different viscosities. At first, the leaflets of the SAVB were stiffened by applying liquid glue (Sekunden Alleskleber Geruchsfrei Easy, UHU, Buehl/Baden, Germany) on the entire leaflet surface. This was followed by the punctual application of a viscous gel glue (Sekundenkleber Blitzschnell Supergel; UHU) on the entire leaflet surface in an eccentric shape, especially across the central section and free edge, to simulate valve thickening and calcific plaques.

During the measurements, diastolic pressure was maintained at $80 \mathrm{~mm} \mathrm{Hg}$; systolic pressure, at $120 \mathrm{~mm} \mathrm{Hg}$; stroke rate, at $64 \mathrm{bpm}$; and stroke volume, at $70 \mathrm{~mL}$. The test solution was a physiological saline 

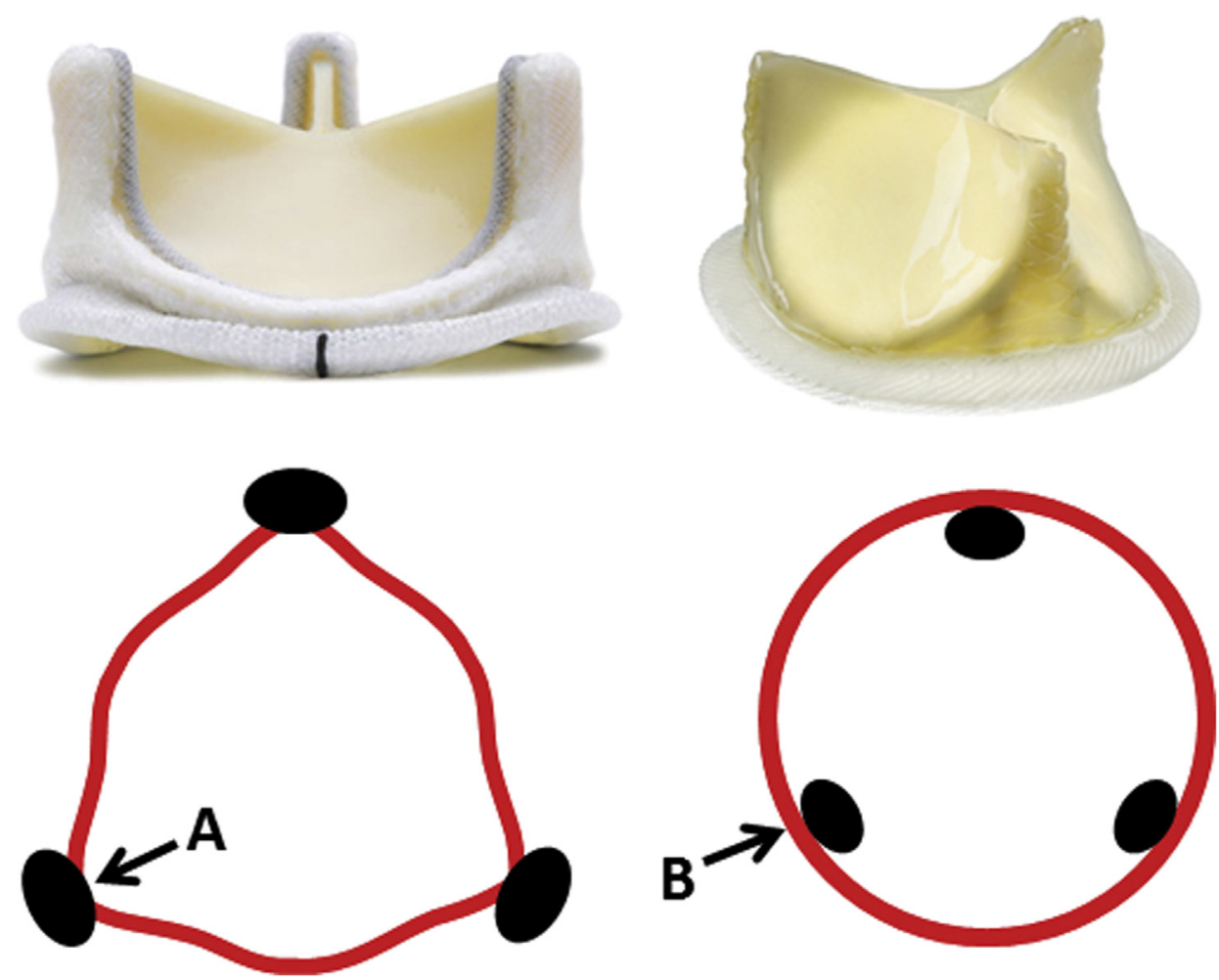

FIGURE 1. Top, The Perimount Magna Ease (left) and Trifecta (right) devices. Bottom, Schematic drawings. The pericardial leaflets (red) are mounted inside the stent frame in the Perimount Magna Ease $(A)$ and outside in the Trifecta $(B)$.

solution $(0.9 \%)$ with a density of $1.0046 \mathrm{~g} / \mathrm{cm}^{3}$ and a dynamic viscosity of $0.9 \mathrm{mPa} \cdot \mathrm{s}$ at an ambient temperature of $20^{\circ} \mathrm{C}$. For coronary flow, the maximum LCF was $120 \mathrm{~mL} /$ minute, and the maximum RCF was $80 \mathrm{~mL} /$ minute. $^{10}$

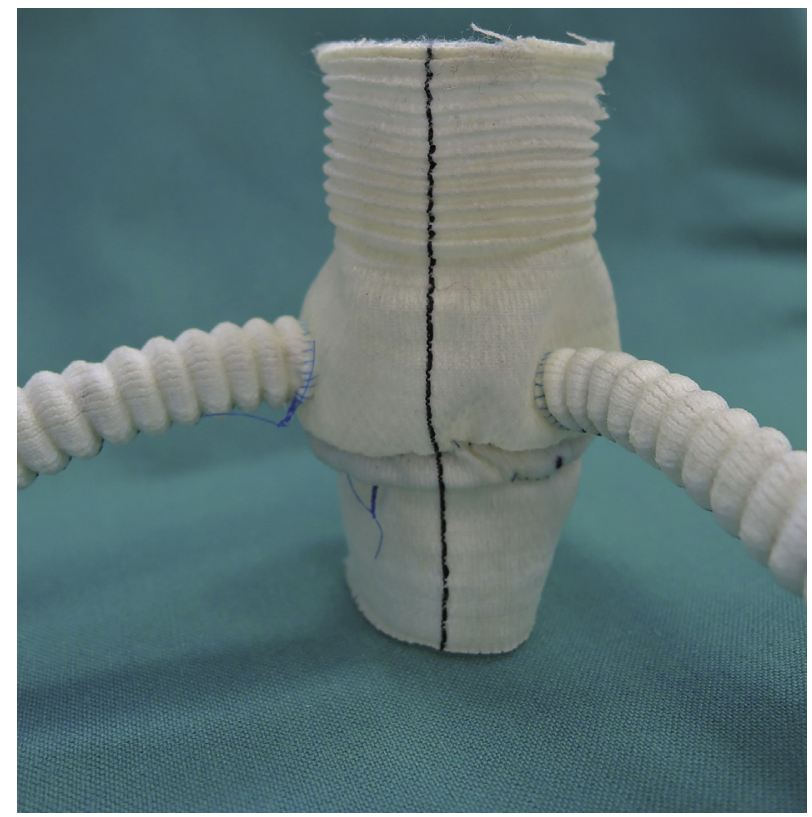

FIGURE 2. An aortic root model. Two Dacron prostheses $(8 \mathrm{~mm}$ diameter), simulating the coronary arteries, are anastomosed with an aortic sinus prosthesis (26 $\mathrm{mm}$ diameter).

\section{Measurement Technique}

Left ventricular pressure ( $4 \mathrm{~cm}$ below the aortic valve) and aortic pressure $(6 \mathrm{~cm}$ above the aortic valve) were measured with 2 capacitive pressure transducers (Envec Ceracore M; Endress+Hauser, Maulburg, Germany), calibrated to a measuring range of -20 to $+160 \mathrm{~mm} \mathrm{Hg}$ and a resolution of $0.02 \mathrm{~mm} \mathrm{Hg}$.

The sensor of an ultrasonic flowmeter (TS-410; Transonic Systems, Ithaca, NY) was mounted directly below the aortic valve to record the volume flow through the valve. The sensor works bidirectionally with a resolution of $2 \mathrm{~mL} /$ minute and records flow rates up to $20 \mathrm{~L} /$ minute. LCF and RCF were measured with a Transonic TS-420 ultrasonic device and 6-mm coronary probes.

A high-speed camera (Motionscope HR-1000; Redlake Imaging, Morgan Hill, Calif) mounted above the conduit recorded the characteristics of motion of the aortic valves with 500 pictures per second. Video recordings and flow measurements were started simultaneously using trigger signals.

\section{Analysis and Statistics}

The values for pressure and flow were registered by an analog-todigital converter recording 500 individual values per second. Each measurement included 10 successive cardiac cycles to calculate the mean value \pm standard deviation. The analysis of the measurement results was based on the international norm for testing cardiac valve prostheses (ISO 5840: cardiovascular implants-cardiac valve prostheses).

The evaluation of GOA was based on photographs taken by the high-speed camera and calculated with ImageJ (National Institutes of Health, Bethesda, Md), using the inner diameter of SAVB as the reference value.

For statistical analyses, the $t$ test or Mann-Whitney $U$ test was applied depending on the given distribution. The level of significance was defined 


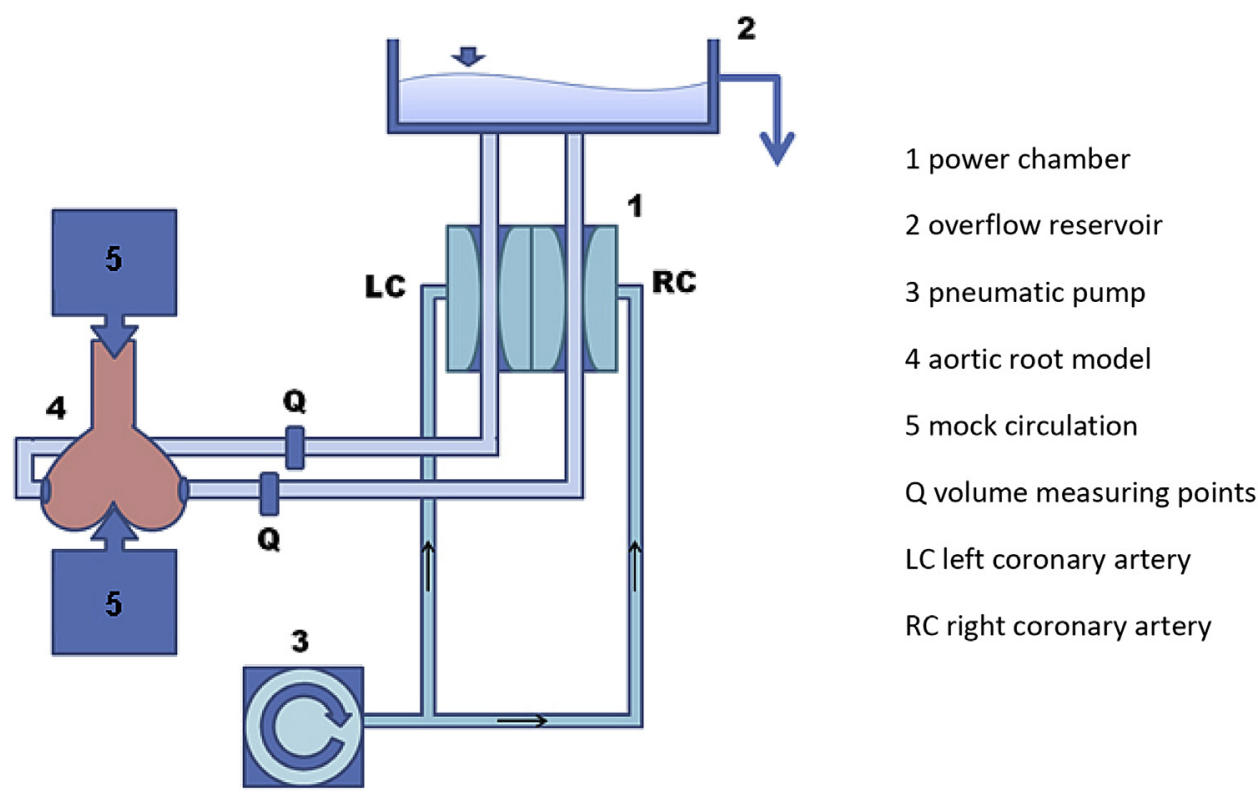

FIGURE 3. Schematic drawing of the coronary flow device. The left coronary artery $(L C)$ and right coronary artery $(R C)$ running out of the aortic root model (4) pass a pressurized power chamber (1). The pressure is regulated by a pneumatic pump (3). The coronary flow is measured at the volume measuring points $(Q)$.

as $P<.05$. Data processing programs $\mathrm{R}$ version 3.1.1 (R Institute for Statistical Computing, Vienna, Austria) and SPSS version 22 (IBM, Armonk, $\mathrm{NY}$ ) were used for statistical evaluation.

\section{RESULTS \\ Coronary Flow}

We found no statistically significant differences in LCF and RCF between the 2 SAVB types before and after TAVI-ViV as well as at the different coronary heights in either the noncalcified model or the calcified model ( $P$ not significant for all) (Table 1$)$.

RCF. The RCF after TAVI-ViV in the noncalcified SAVB dropped by $7 \%$ at a coronary height of $8 \mathrm{~mm}$ and by $9 \%$ at a coronary height of $10 \mathrm{~mm}$ with the Trifecta and by $2 \%$ at a coronary height of $8 \mathrm{~mm}$ and $3 \%$ at a coronary height of $10 \mathrm{~mm}$ with the Perimount Magna Ease. In the calcified SAVB model, coronary flow after TAVI-ViV decreased by $8 \%$ at a coronary height of $8 \mathrm{~mm}$ and $10 \%$ at a coronary height of $10 \mathrm{~mm}$ with the Trifecta, and increased by $6 \%$ at a coronary height of $8 \mathrm{~mm}$ and remained unchanged at a coronary height of $10 \mathrm{~mm}$ with the Perimount Magna Ease.

LCF. After TAVI-ViV in the noncalcified model, LCF decreased by $3 \%$ at a coronary height of $8 \mathrm{~mm}$ and by $9 \%$ at a coronary height of $10 \mathrm{~mm}$ with the Trifecta and remained equal at a coronary height of $8 \mathrm{~mm}$ and dropped by $5 \%$ at a coronary height of $10 \mathrm{~mm}$ with the Perimount Magna Ease. In the calcified model, LCF decreased by $8 \%$ at a coronary height of $8 \mathrm{~mm}$ and by $4 \%$ at a coronary height of $10 \mathrm{~mm}$ with the Trifecta and decreased by $8 \%$ at a coronary height of $8 \mathrm{~mm}$ but increased by $6 \%$ at a coronary height of $10 \mathrm{~mm}$ with the Perimount Magna Ease.

\section{GOA}

Noncalcified model. With both the Trifecta and in Perimount Magna Ease, TAVI-ViV resulted in a decrease in GOA $(P<.001$ for both), with a significant difference between the 2 SAVB types $(P<.001)$ (Table 2$)$.

Calcified model. GOA increased after TAVI-ViV with both the Trifecta and the Perimount Magna Ease $(P<.001$ for both). There was no statistically significant difference between the 2 types ( $P$ not significant) (Table 2 ).

\section{Pressure Gradients}

Noncalcified model. With both the Trifecta and the Perimount Magna Ease, TAVI-ViV resulted in increases in $\mathrm{dP}_{\text {max }}(P$ not significant for both $)$ and $\mathrm{dP}_{\text {mean }}(P=.0001$ and .006 , respectively). There were statistically significant differences between the SAVB types in $\mathrm{dP}_{\text {mean }}$ before TAVI-ViV $(P=.009)$ and in $\mathrm{dP}_{\max }$ after TAVI-ViV $(P=.036)$ (Table 2).

Calcified model. $\mathrm{dP}_{\max }$ and $\mathrm{dP}_{\text {mean }}$ values decreased after TAVI-ViV with both the Trifecta and the Perimount Magna Ease $\left(\mathrm{dP}_{\max }, P=.0003\right.$ and .0004 , respectively; $\mathrm{dP}_{\text {mean }}$, $P<.001$ for both). There were no significant differences in either parameter between the 2 types of SAVB ( $P$ not significant) (Table 2).

\section{Overlapping of Trifecta Leaflets}

Implantation of the Sapien XT THV with the Trifecta SAVB resulted in an overlapping of the Trifecta leaflets around and above the THV (Figure 5). This overlap occurred in all TAVI-ViV procedures during our experiments, in both the noncalcified and calcified models. 


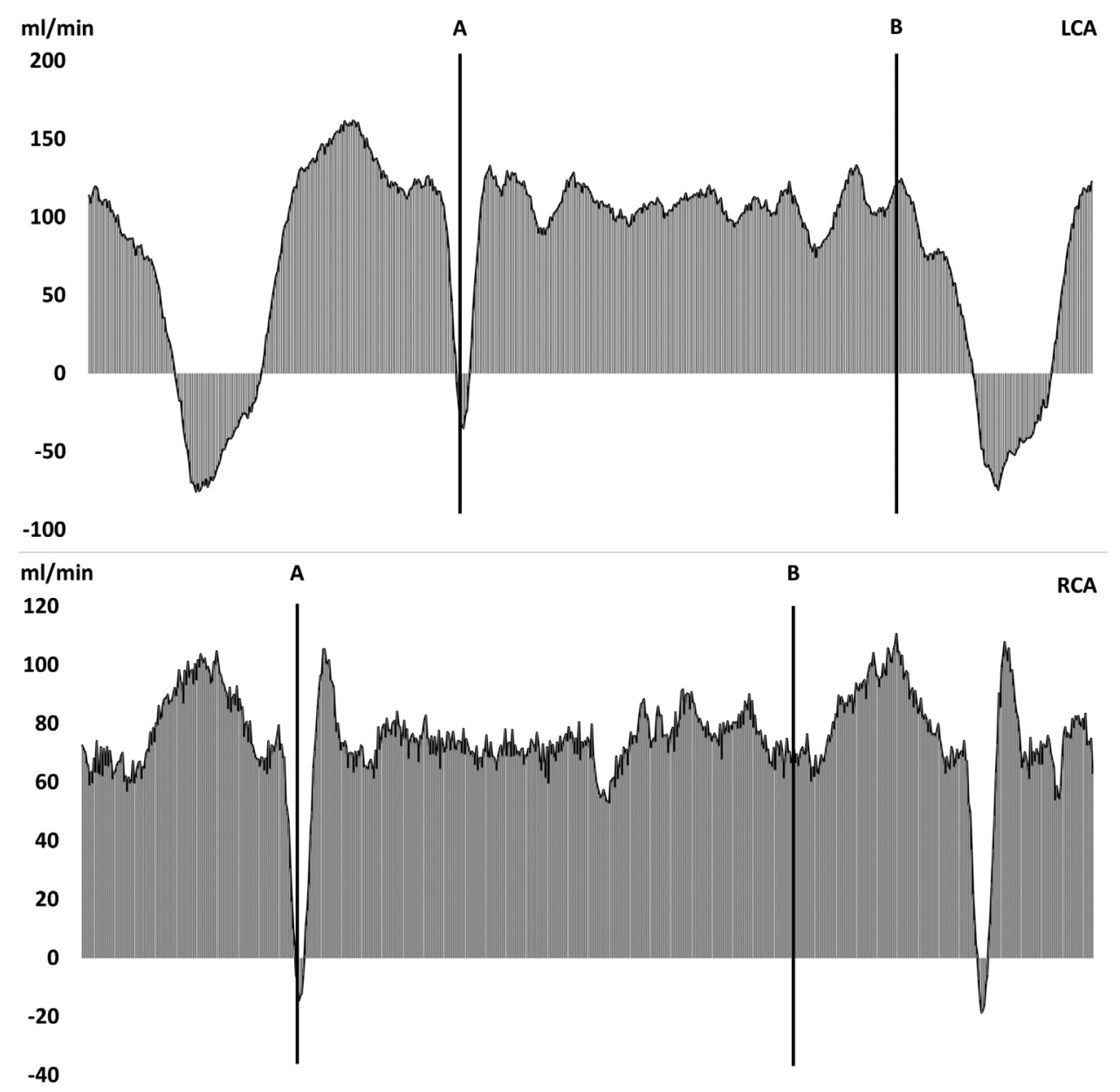

FIGURE 4. Left (top) and right (bottom) coronary flow curves measured during an experimental run. For the statistical analysis in this study, the coronary flow during diastole was used (area between $A$ and $B$ ).

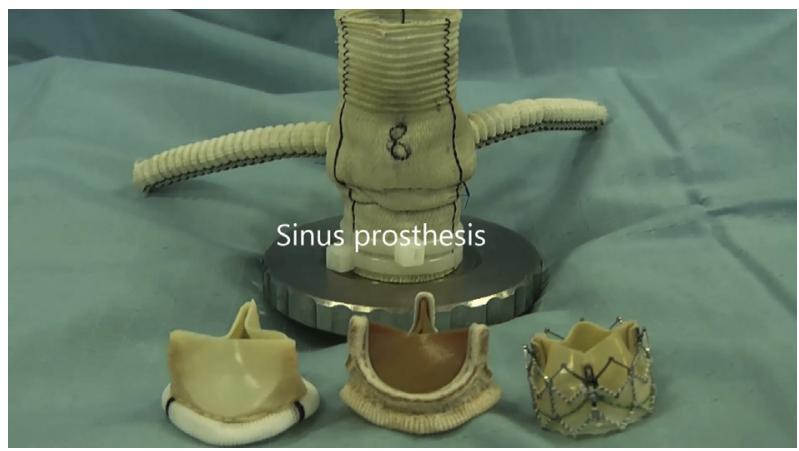

VIDEO 1. The experimental procedure of our in vitro study. A Trifecta surgical aortic valve bioprosthesis, size 25, was implanted into the aortic root model with a coronary ostia height of $8 \mathrm{~mm}$, and the conduit was inserted into the mock circulation. Then transcatheter aortic valve-invalve implantation with a Sapien XT transcatheter heart valve, size 23, was performed, and the conduit was reinserted into the mock circulation likewise. Video available at: http://www.jtcvsonline.org/article/S00225223(16)31485-4/addons.

\section{DISCUSSION}

\section{Coronary Flow}

This in vitro study provides no evidence of a significant difference in coronary flow after TAVI-ViV in SAVBs with externally mounted leaflet tissue (Trifecta) and internally mounted leaflet tissue (Perimount Magna Ease). Several factors are known to increase the risk of coronary obstruction. Besides predisposing anatomic aspects, such as a low coronary ostia height or a narrow aortic root and sinotubular junction, the characteristics of the SAVB must be considered. Risk factors for coronary obstruction include a supra-annular implantation, a high profile of the SAVB, and the SAVB's locational relationship to the coronary ostia. ${ }^{1,11,12}$ Based on clinical studies, Dvir et $\mathrm{al}^{2,3}$ hypothesized that coronary obstruction occurs preferentially in modern SAVBs with externally mounted leaflet tissue, such as the Trifecta. These authors also found that coronary obstruction was more frequent in patients with a calcified SAVB compared with patients 

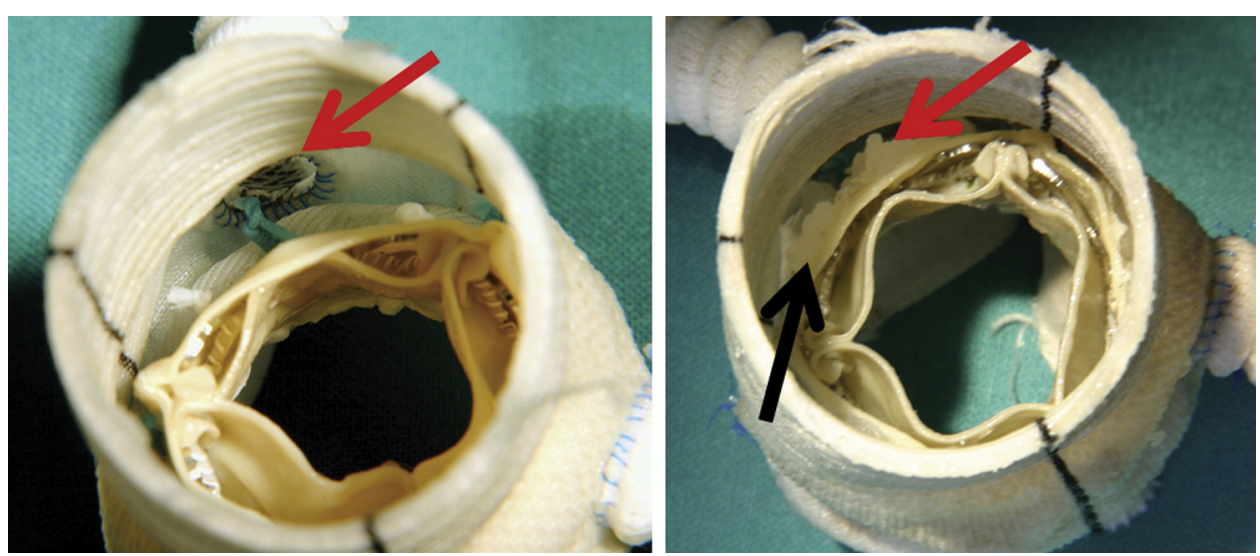

FIGURE 5. Aortic root model with valve-in-valve implantation of a Sapien XT in a noncalcified (left) and calcified (right) Trifecta SAVB. The red arrows indicate the remaining space between leaflet tissue and coronary ostium. This space is obviously reduced in the calcified model. The black arrow indicates the calcification of a Trifecta, simulated with glue (see Materials and Methods).

with a degenerated regurgitant SAVB. ${ }^{13}$ As the main reason for this fatal complication, they suggested the displacement of calcified and thickened tissue close to the coronary ostia. $^{14}$

In the present in vitro study, we aimed to investigate further the hypotheses of Dvir et al to improve the treatment of patients with degenerated SAVBs and to identify an increased risk of coronary obstruction. Our findings do not verify these hypotheses, however. A critical obstruction of coronary flow after TAVI-ViV did not occur in SAVBs with internally mounted or externally mounted leaflets in our models. In both experimental runs-the noncalcified and calcified models-even with a low coronary ostia, TAVI-ViV did not cause any significant change in coronary flow profile. The distance between the coronary ostia and the SAVB leaflet tissue remained within the range of the

TABLE 1. Coronary flow

\begin{tabular}{|c|c|c|c|c|}
\hline Parameter & TRI & $\begin{array}{c}\text { TRI }+ \\
\text { TAVI-ViV }\end{array}$ & PERI & $\begin{array}{c}\text { PERI + } \\
\text { TAVI-ViV }\end{array}$ \\
\hline \multicolumn{5}{|l|}{$\mathrm{RCF}, \mathrm{mL} / \mathrm{stroke}$} \\
\hline \multicolumn{5}{|l|}{ Noncalcified } \\
\hline CH 8 mm & $0.64 \pm 0.06$ & $0.60 \pm 0.07$ & $0.62 \pm 0.08$ & $0.61 \pm 0.07$ \\
\hline $\mathrm{CH} 10 \mathrm{~mm}$ & $0.62 \pm 0.06$ & $0.58 \pm 0.04$ & $0.62 \pm 0.04$ & $0.60 \pm 0.06$ \\
\hline \multicolumn{5}{|l|}{ Calcified } \\
\hline CH 8 mm & $0.67 \pm 0.05$ & $0.62 \pm 0.04$ & $0.65 \pm 0.06$ & $0.69 \pm 0.17$ \\
\hline CH $10 \mathrm{~mm}$ & $0.64 \pm 0.08$ & $0.58 \pm 0.06$ & $0.58 \pm 0.11$ & $0.58 \pm 0.03$ \\
\hline \multicolumn{5}{|l|}{ LCF, mL/stroke } \\
\hline \multicolumn{5}{|l|}{ Noncalcified } \\
\hline $\mathrm{CH} 8 \mathrm{~mm}$ & $0.90 \pm 0.08$ & $0.87 \pm 0.06$ & $0.89 \pm 0.06$ & $0.89 \pm 0.09$ \\
\hline CH $10 \mathrm{~mm}$ & $0.89 \pm 0.05$ & $0.82 \pm 0.07$ & $0.87 \pm 0.04$ & $0.83 \pm 0.03$ \\
\hline \multicolumn{5}{|l|}{ Calcified } \\
\hline CH 8 mm & $0.85 \pm 0.06$ & $0.79 \pm 0.08$ & $0.83 \pm 0.04$ & $0.77 \pm 0.12$ \\
\hline $\mathrm{CH} 10 \mathrm{~mm}$ & $0.79 \pm 0.11$ & $0.76 \pm 0.04$ & $0.76 \pm 0.13$ & $0.80 \pm 0.04$ \\
\hline
\end{tabular}

aortic root models used in this study; however, in the calcified model, this space was reduced (Figure 5). It is conceivable that with an increasing amount of calcific deposits, this space could be reduced further, causing coronary obstruction. Nevertheless, TAVI-ViV with both modern SAVB types has proven feasible, and the risk of coronary obstruction can be estimated similarly in patients with anatomy like the geometry of our aortic root models.

Careful, detailed preintervention planning seems to be a prerequisite for minimizing the risk of coronary obstruction in general. ${ }^{1} \mathrm{~A}$ cardiac computed tomography scan before the TAVI-ViV procedure provides exact images of the patient's anatomy. Apart from aortic root characteristics, such as diameter and height of the sinuses and the sinotubular junction, as well as coronary ostia height, the characteristics and radiologic appearance of the degenerated SAVB should be considered. ${ }^{15}$ In addition, 3D-echocardiography with aortography is recommended. ${ }^{12}$ These recommendations are supported by the studies reported by Gurvitch et al ${ }^{4}$ and Ye et al. ${ }^{5}$ According to these

TABLE 2. Hydrodynamics

\begin{tabular}{|c|c|c|c|c|}
\hline Parameter & TRI & $\begin{array}{c}\text { TRI + } \\
\text { TAVI-ViV } \\
\end{array}$ & PERI & $\begin{array}{c}\text { PERI + } \\
\text { TAVI-ViV }\end{array}$ \\
\hline \multicolumn{5}{|l|}{ Geometric orifice area } \\
\hline Noncalcified, $\mathrm{cm}^{2}$ & $2.48 \pm 0.05$ & $1.43 \pm 0.12$ & $2.08 \pm 0.03$ & $1.56 \pm 0.17$ \\
\hline Calcified, $\mathrm{cm}^{2}$ & $0.94 \pm 0.32$ & $1.3 \pm 0.14$ & $0.77 \pm 0.36$ & $1.42 \pm 0.17$ \\
\hline \multicolumn{5}{|l|}{ Pressure gradients } \\
\hline \multicolumn{5}{|l|}{ Noncalcified } \\
\hline $\mathrm{dP}_{\max }, \mathrm{mm} \mathrm{Hg}$ & $12 \pm 2$ & $13 \pm 2$ & $13 \pm 2$ & $14 \pm 2$ \\
\hline $\mathrm{dP}_{\text {mean }}, \mathrm{mm} \mathrm{Hg}$ & $4 \pm 1$ & $5 \pm 1$ & $5 \pm 1$ & $6 \pm 1$ \\
\hline \multicolumn{5}{|l|}{ Calcified } \\
\hline $\mathrm{dP}_{\max }, \mathrm{mm} \mathrm{Hg}$ & $23 \pm 7$ & $16 \pm 1$ & $34 \pm 20$ & $15 \pm 2$ \\
\hline $\mathrm{dP}_{\text {mean }}, \mathrm{mm} \mathrm{Hg}$ & $12 \pm 5$ & $6 \pm 1$ & $20 \pm 13$ & $6 \pm 1$ \\
\hline
\end{tabular}

Data are mean \pm standard deviation. TRI, Trifecta; TAVI-ViV, transcatheter aortic valve-in-valve implantation; $P E R I$, Perimount Magna Ease; $d P$, pressure gradient. 
authors, the major influences on coronary perfusion are the type of SAVB used and also precise preintervention planning to avoid coronary occlusion. This idea is in alignment with the findings of Haussig et al, ${ }^{16}$ who reported a successful TAVI-ViV with a modern SAVB (Trifecta, size 23) without coronary obstruction, using precise preintervention imaging.

Risk factors for coronary obstruction identified on preintervention imaging are not necessarily considered an absolute contraindication for TAVI-ViV, however. Several strategies for avoiding coronary occlusion are available. Intraoperative transesophageal echocardiography and angiography enable rapid detection of coronary occlusion. Intraprocedural balloon inflation is a useful technique for estimating the risk of coronary obstruction during the TAVI-ViV procedure. A balloon of similar size as the intended THV results in temporary displacement of the SAVB leaflets and reveals by means of aortography the remaining space between the coronary ostia and SAVB leaflets. ${ }^{17}$ The intraoperative placement of a guide wire in the coronary artery at risk seems to help prevent dislocation of leaflet tissue, as well as ensure emergency access to the coronary artery. ${ }^{12,14,18,19}$ Dvir et $\mathrm{al}^{2}$ even recommended the implantation of a smaller transcatheter heart valve to reduce the risk of coronary obstruction. This "downsizing" approach has been supported by further in vitro investigations by our group. ${ }^{20}$ In addition, the implantation of a low-profile, retrievable, and repositionable THV, such as the Lotus (Boston Scientific, Marlborough, Mass) or Direct Flow (Direct Flow Medical, Santa Rosa, Calif) THV, appears to provide a viable option for avoiding or dealing with coronary obstruction. Successful TAVI-ViV procedures with these THVs have been reported. ${ }^{21,22}$ In cases where coronary obstruction is detected, these THVs can be retrieved and implanted in a new position. This technique is supported by Wolf et $\mathrm{al}^{23}{ }^{23}$ who reported successful repositioning of a Direct Flow THV due to right coronary occlusion. In cases involving a transapical approach for TAVI-ViV, the JenaValve (JenaValve Technology, Irvine, Calif) might be a valuable option to avoid coronary occlusion. The unique anchoring mechanism of this THV fixes the leaflets of the SAVB, possibly preventing leaflet dislocation to the coronary ostia.

The reliability of these strategies remains uncertain, however, owing to the small patient numbers in the foregoing studies. Thus, in patients with the aforementioned risk factors for coronary obstruction, a classical surgical approach may be considered.

\section{GOA}

In the noncalcified model, TAVI-ViV led to a significant reduction in GOA, owing to the size difference between the SAVB (size 25) and the THV (size 23). There was a significant difference in GOA between the Trifecta and the Perimount Magna Ease SAVBs, with the externally mounted leaflets of the Trifecta allowing for a greater GOA than is allowed by the geometry of a Perimount Magna Ease of identical size. ${ }^{6}$ This observation is important with regard to TAVI-ViV procedures in degenerated SAVBs without stenosis. In this case, regurgitation is treated successfully, but at the cost of a considerable reduction in GOA. This fact should be taken into account during preinterventional planning. Ruel et $\mathrm{al}^{24}$ supported this recommendation by suggesting a correlation between a small GOA and a significantly higher rate of postprocedure heart failure.

TAVI-ViV in calcified SAVBs resulted in increases in GOA. The artificial calcification of the SAVB caused a reduction in GOA compared with the noncalcified model and nullified the geometrical discrepancy between the Trifecta and Perimount Magna Ease SAVBs. Thus, there was no significant difference in GOA by SAVB type.

\section{Pressure Gradients}

In the noncalcified model, TAVI-ViV was associated with a significant increase in $\mathrm{dP}_{\text {mean }}$, owing to the reduction in GOA mentioned above. The results of this experimental run showed a significant difference between the Trifecta and Perimount Magna Ease SAVBs, related to the lower pressure gradients in the Trifecta compared with a Perimount Magna Ease of the same size. ${ }^{6}$ Inevitably, TAVI-ViV with the Trifecta causes a greater increase in pressure gradients than occurs with the Perimount Magna Ease. According to Ruel et al, ${ }^{24}$ this is clinically important because of the linear relationship between increased pressure gradients and development of heart failure.

In the calcified model, TAVI-ViV decreased $\mathrm{dP}_{\max }$ and $\mathrm{dP}_{\text {mean }}$ significantly. The effect of SAVB type could not be evaluated, because the artificial calcification nullified the original geometric characteristics of the Trifecta and Perimount Magna Ease SAVBs. The clinical relevance of this reduction in pressure gradients after TAVI-ViV is supported by the findings of Chan et al, ${ }^{25}$ who reported that a reduction in pressure gradients led to a significantly lower incidence of heart failure in a selected patient cohort compared with patients with unchanged pressure gradients. ${ }^{25}$

\section{Overlapping of Trifecta Leaflets}

Another finding during our experiments was an overlapping of the Trifecta leaflets around and above the Sapien XT THV in both the noncalcified and calcified models (Figure 5). This finding was similar in all tests and was likely caused by the high profile of the Trifecta. This overlapping of the Trifecta leaflets may provide a barrier to forward flow and lead to pressure gradients, which are not caused by the implanted THV. Furthermore, turbulence may occur downstream of the overlapping 
leaflets, potentially creating an additional risk of thrombus formation behind the Trifecta with unknown consequences. The overlapping of leaflet tissue might be avoided by implanting a THV with a higher stent frame compared with the Sapien XT, for example, the EvolutR (Medtronic, Minneapolis, Minn).

\section{Limitations}

Valves. Only 2 Sapien XT devices were available for the execution of 40 TAVI-ViV procedures; thus, each valve needed to be crimped and dilated as well as explanted from the SAVB several times, leading to increasing deformation of the THV.

Valve calcification. The artificial calcification using glue is only an approximation to leaflet calcification in vivo. Although the leaflets were stiffened and thickened, transvalvular gradients remained smaller than one would expect in severe aortic valve stenosis. This is likely related to the specific material properties of the glue, which might be less rigid than actual calcification. Another limitation is the fact that we did not study the effect of the location and the shape of leaflet calcification and the variations in leaflet height, both of which may be potential risk factors for coronary obstruction.

Aortic root models. In contrast to the human aorta, the aortic root models lacked elasticity; thus, there was no alteration of its diameter during systole and diastole. Furthermore, the sinuses of the aortic root models were all the same size, but in vivo, the right coronary sinus is larger in size than the left and noncoronary sinuses. This may lead to a more frequent obstruction of the left coronary ostium compared with obstruction of the right coronary ostium. We also simulated only a single size of the aortic bulge, but human aortic roots with the same size of the annulus also show smaller sinuses sizes than seen in aortic root models. ${ }^{26}$ This anatomy was seen in a patient suffering from coronary obstruction after TAVI-ViV. ${ }^{1}$

\section{CONCLUSIONS}

The supposition of an SAVB with externally mounted leaflets (Trifecta) decreasing coronary flow pathologically could not be verified. In principle, TAVI-ViV in SAVBs with externally mounted or internally mounted leaflets is a feasible treatment option for patients with a degenerated SAVB and an aortic root diameter identical to that in our limited model. Nonetheless, detailed preinterventional planning concerning the individual anatomy of the aortic root and the characteristics of the SAVB is essential given the wide variety of pathologies.

\section{Conflict of Interest Statement}

S.S., T.H., E.I.C., D.R., and H.-H.S. have received travel grants from St Jude Medical and Edwards Lifesciences. E.I.C. holds significant stock in Edwards Lifesciences.
T.H. is a consultant for St Jude Medical. All other authors have nothing to disclose with regard to commercial support.

We thank Michael Diwoky and Tobias Frin for their excellent data management and analyses and for their assistance in preparing this manuscript for publication.

\section{References}

1. Gurvitch R, Cheung A, Bedogni F, Webb JG. Coronary obstruction following transcatheter aortic valve-in-valve implantation for failed surgical bioprostheses. Catheter Cardiovasc Interv. 2011;77:439-44.

2. Dvir D, Webb J, Brecker S, Bleiziffer S, Hildick-Smith D, Colombo A, et al. Transcatheter aortic valve replacement for degenerative bioprosthetic surgical valves: results from the global valve-in-valve registry. Circulation. 2012;126: 2335-44.

3. Dvir D, Barbanti M, Tan J, Webb JG. Transcatheter aortic valve-in-valve implantation for patients with degenerative surgical bioprosthetic valves. Curr Probl Cardiol. 2014;39:7-27.

4. Gurvitch R, Cheung A, Ye J, Wood DA, Willson AB, Toggweiler S, et al. Transcatheter valve-in-valve implantation for failed surgical bioprosthetic valves. J Am Coll Cardiol. 2011;58:2196-209.

5. Ye J, Webb JG, Cheung A, Soon JL, Wood D, Thompson CR, et al. Transapical transcatheter aortic valve-in-valve implantation: clinical and hemodynamic outcomes beyond 2 years. J Thorac Cardiovasc Surg. 2013;145: 1554-62.

6. Wendt D, Thielmann M, Plicht B, Aßmann J, Price V, Neuhäuser M, et al. The new St Jude Trifecta versus Carpentier-Edwards Perimount Magna and Magna Ease aortic bioprosthesis: is there a hemodynamic superiority? J Thorac Cardiovasc Surg. 2014;147:1553-60.

7. Ferrari E. Transapical aortic "valve-in-valve" procedure for degenerated stented bioprosthesis. Eur J Cardiothorac Surg. 2012;41:485-90.

8. Ribeiro HB, Webb JG, Makkar RR, Cohen MG, Kapadia SR, Kodali S, et al. Predictive factors, management, and clinical outcomes of coronary obstruction following transcatheter aortic valve implantation: insights from a large multicenter registry. J Am Coll Cardiol. 2013;62:1552-62.

9. Scharfschwerdt M, Misfeld M, Sievers HH. The influence of a nonlinear resistance element upon in vitro aortic pressure tracings and aortic valve motions. ASAIO J. 2004;50:498-502.

10. Transonic Systems Inc. Physiology of graft flow. In: Eymann S, ed. The CABG Handbook. 2nd ed. Ithaca, NY: Transonic Systems Inc; 2014:52-8.

11. Russ C, Hopf R, Hirsch S, Sündermann S, Falk V, Székely G, et al. Simulation of transcatheter aortic valve implantation under consideration of leaflet calcification. Conf Proc IEEE Eng Med Biol Soc. 2013;2013:711-4.

12. Webb JG, Dvir D. Transcatheter aortic valve replacement for bioprosthetic aortic valve failure: the valve-in-valve procedure. Circulation. 2013;127:2542-50.

13. Dvir D, Webb JG, Bleiziffer S, Pasic M, Waksman R, Kodali S, et al. Transcatheter aortic valve implantation in failed bioprosthetic surgical valves. JAMA. 2014;312:162-70.

14. Kapadia SR, Svensson L, Tuzcu EM. Successful percutaneous management of left main trunk occlusion during percutaneous aortic valve replacement. Catheter Cardiovasc Interv. 2009;73:966-72.

15. Bapat V, Mydin I, Chadalavada S, Tehrani H, Attia R, Thomas M. A guide to fluoroscopic identification and design of bioprosthetic valves: a reference for valve-in-valve procedure. Catheter Cardiovasc Interv. 2013;81: 853-61.

16. Haussig S, Schuler G, Linke A. Treatment of a failing St Jude Medical Trifecta by Medtronic Corevalve Evolut valve-in-valve implantation. JACC Cardiovasc Interv. 2014;7:e81-2.

17. Dvir D, Leipsic J, Blanke P, Ribeiro HB, Kornowski R, Pichard A, et al. Coronary obstruction in transcatheter aortic valve-in-valve implantation: preprocedural evaluation, device selection, protection, and treatment. Circ Cardiovasc Interv. 2015;8:e002079.

18. Chakravarty T, Jilaihawi H, Nakamura M, Kashif M, Kar S, Cheng W, et al. Preemptive positioning of a coronary stent in the left anterior descending artery for left main protection: a prerequisite for transcatheter aortic valve-in-valve implantation for failing stentless bioprostheses? Catheter Cardiovasc Interv. 2013;82:E630-6.

19. Urena M, Nombela-Franco L, Doyle D, De Larochellière R, Dumont E, Villeneuve J, et al. Transcatheter aortic valve implantation for the treatment of 
surgical valve dysfunction ("valve-in-valve"): assessing the risk of coronary obstruction. J Card Surg. 2012;27:682-5.

20. Stock S, Scharfschwerdt M, Meyer-Saraei R, Richardt D, Charitos EI, Sievers HH, et al. Does undersizing of transcatheter aortic valve bioprostheses during valve-in-valve implantation avoid coronary obstruction? An in vitro study. Thorac Cardiovasc Surg. June 15, 2016 [Epub ahead of print].

21. Conradi L, Silaschi M, Seiffert M, Lubos E, Blankenberg S, Reichenspurner H, et al. Transcatheter valve-in-valve therapy using 6 different devices in 4 anatomic positions: clinical outcomes and technical considerations. J Thorac Cardiovasc Surg. 2015;150:1557-65. 1567.e1-3; discussion 1565-7.

22. Castriota F, Cavazza C, Secco GG, Micari A, Cremonesi A. First Lotus aortic valve-in-valve implantation to treat degenerated Mitroflow bioprostheses. EuroIntervention. 2016;11:1545-8.
23. Wolf A, Schmitz T, Latib A, Naber CK. Successful repositioning of a direct-flow medical 25-mm valve due to acute occlusion of right coronary artery during transcatheter aortic valve replacement procedure. JACC Cardiovasc Interv. 2015;8:e33-4.

24. Ruel M, Rubens FD, Masters RG, Pipe AL, Bédard P, Mesana TG. Late incidence and predictors of persistent or recurrent heart failure in patients with mitral prosthetic valves. J Thorac Cardiovasc Surg. 2004;128: 278-83.

25. Chan V, Rubens F, Boodhwani M, Mesana T, Ruel M. Determinants of persisten or recurrent congestive heart failure after contemporary surgical aortic valve replacement. J Heart Valve Dis. 2014;23:665-70.

26. Berdajs D, Lajos P, Turina M. The anatomy of the aortic root. Cardiovasc Surg. 2002;10:320-7.

Readers who found these articles interesting may also like to read the following papers found in recent and future issues of our sister publications, Seminars in Thoracic and Cardiovascular Surgery and Operative Techniques in Thoracic and Cardiovascular Surgery!

\section{Acquired: Valvular Heart Disease}

Original Submission: Should Moderate-to-Severe Tricuspid Regurgitation be Repaired During Reoperative Left-Sided Valve Procedures? Igor Gosev. Semin Thoracic Surg 2016; 28:38-45.

Editorial Commentary: To Fix or Not to Fix? That is the Question. Kenton J. Zehr. Semin Thoracic Surg 2016;28:46-47.

Original Submission: Current Hypotheses in Cardiac Surgery: Biofilm in Infective Endocarditis. Haytham Elgharably. Semin Thoracic Surg 2016; 28:56-59.

Editorial Commentary: Treatment of Infective Endocarditis - Are We On the Right Track? Hans-Joachim Schafers. Semin Thoracic Surg 2016; 28:60-61.

Original Submission: Posterior Leaflet Detachment, Augmentation, and Reconstruction for Treatment of Functional Mitral Valve Regurgitation. Rakesh M. Suri. Semin Thoracic Surg 2015; 27:91-94.

Editorial Commentary: Patch Augmentation of Mitral Valve Leaflet in Ischemic Mitral Regurgitation. Tirone E. David. Semin Thoracic Surg 2015; 27:95-96.

Original Submission: Elevated Stroke Risk Associated with Femoral Artery Cannulation During Mitral Valve Surgery. Kareem Bedeir. Semin Thoracic Surg 2015; 27:97-103.

Editorial Commentary: Are We Going Backwards or Forwards in Minimally Invasive Mitral Valve Surgery? Three Eras of Perfusion Strategy. Vinay Badhwar. Semin Thoracic Surg 2015; 27:104-105.

Current Readings: Selection of Valve Prostheses. Cristian Rosu. Semin Thoracic Surg 2015; 27:152-158.

Original Submission: Long-Term Hemodynamic Performance of the Aortic Valve Late After David I: An Echocardiographic Study. Antonino S. Rubino. Semin Thoracic Surg 2015; 27:257-263.

Editorial Commentary: Back to David I. Tirone E. David. Semin Thoracic Surg 2015; 27:264-265.

Discussion in Cardiothoracic Treatment and Care: Aortic Valve/Root Repair. Tirone David. Semin Thoracic E Surg 2015; 27:271287.

Biatrial Transseptal Approach for Combined Mitral Valve and Tricuspid Valve Operations. Mahim Malik. Oper Tech Thorac Cardiovasc Surg 2015; 20:2-16.

Mitral Valve Replacement for Infective Endocarditis with Annular Abscess: Annular Reconstruction. Gregory J. Bittle. Oper Tech Thorac Cardiovasc Surg 2015;20:17-30.

Mitral Annuloplasty Using A Biodegradable Annuloplasty Ring. Sanjay Cherian. Oper Tech Thorac Cardiovasc Surg 2015; 20:124134.

Myxomatous Mitral Valve Repair: Loop Neochord Technique. Robert C. Neely. Oper Tech Thorac Cardiovasc Surg 2015; 20:106-123. 


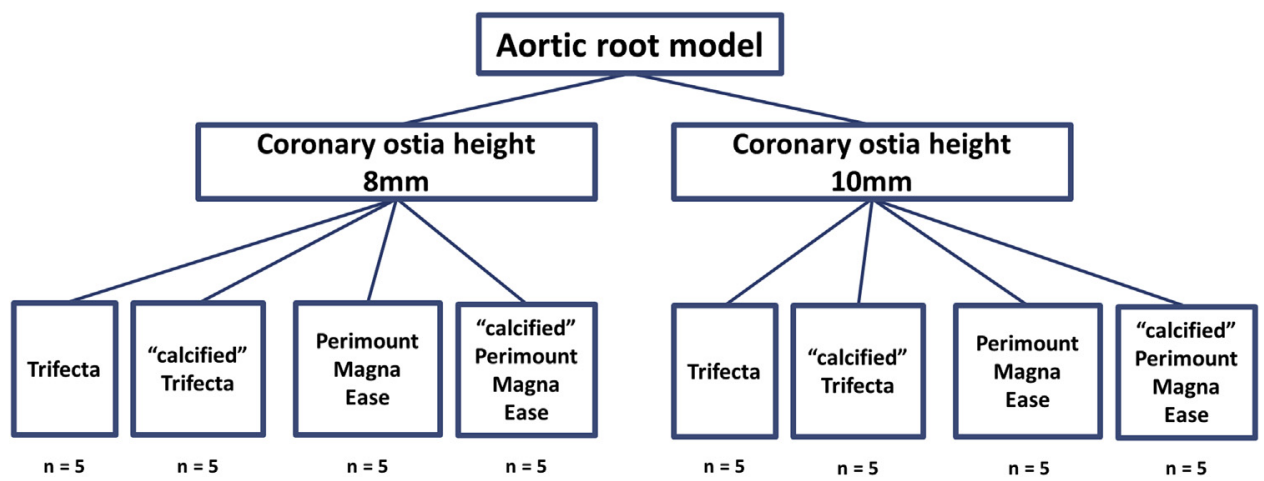

FIGURE E1. Flow chart of the test protocol. In each aortic root model, the different types of bioprostheses were implanted, followed by transcatheter aortic valve-in-valve implantation. 